\title{
High diagnostic accuracy of automated rapid Strep A test reduces antibiotic prescriptions for children in the United Arab Emirates
}

Salama Bin Hendi ${ }^{1}$, Zainab A. Malik ${ }^{1,2,3^{*}}$ (D, Amar Hassan Khamis ${ }^{1,4}$ and Fadil Y. A. Al-Najjar ${ }^{1,2}$

\begin{abstract}
Background: Diagnosis of Group A Streptococcus (GAS) pharyngitis in children is hindered by variable sensitivity of clinical criteria and rapid Strep A tests (SAT), resulting in reliance on throat cultures as the gold standard for diagnosis. Delays while awaiting culture reports result in unnecessary antibiotic prescriptions among children, contributing to the spread of antimicrobial resistance (AMR).

Methods: Diagnostic accuracy study of an automated SAT (A-SAT) in children up to 16 years of age presenting to an emergency room with signs and symptoms of pharyngitis between March and June 2019. Paired throat swabs for A-SAT and culture were collected. Sensitivity, specificity, positive predictive value (PPV) and negative predictive value (NPV) for A-SAT were calculated.

Results: Two hundred and ninety-one children were included in this study. 168 (57.7\%) were boys and the mean age was 4.2 years. A-SAT was positive in 94 (32.3\%) and throat culture was positive in 90 (30.9\%) children. A-SAT and throat culture results showed a high level of consistency in our cohort. Only 6 (2\%) children had inconsistent results, demonstrating that the A-SAT has a high sensitivity (98.9\%), specificity (97.5\%), PPV (94.7\%) and NPV (99.5\%) for the diagnosis of GAS pharyngitis in children. Only 92 (32\%) children were prescribed antibiotics while the vast majority (68\%) were not.

Conclusions: A-SAT is a quick and reliable test with diagnostic accuracy comparable to throat culture. Its widespread clinical use can help limit antibiotic prescriptions to children presenting with pharyngitis, thus limiting the spread of AMR.
\end{abstract}

Keywords: Pharyngitis, Children, Rapid strep, Throat culture, Diagnostic accuracy, Antibiotics, Streptococcus pyogenes

*Correspondence: Zainab_akhtar_malik@hotmail.com;

Zainab.malik@mediclnic.ae

${ }^{1}$ College of Medicine, Mohamed Bin Rashid University of Medicine and Health Sciences, Dubai Healthcare City, Building 14, Dubai 505005, United Arab Emirates

${ }^{2}$ Department of Pediatrics, Mediclinic City Hospital. Dubai Healthcare City, Building 37, Dubai 505004, United Arab Emirates

Full list of author information is available at the end of the article

\section{Background}

Antimicrobial resistance (AMR) is a rapidly-growing global health threat [1]. Infections caused by antibioticresistant pathogens increase the burden of both healthcare-associated and community-acquired infections, leading to increased healthcare utilization and high mortality [2, 3]. Inappropriate antibiotic use in community and healthcare settings is a major driver of antibiotic resistance worldwide [4].

(c) The Author(s). 2021 Open Access This article is licensed under a Creative Commons Attribution 4.0 International License, which permits use, sharing, adaptation, distribution and reproduction in any medium or format, as long as you give appropriate credit to the original author(s) and the source, provide a link to the Creative Commons licence, and indicate if changes were made. The images or other third party material in this article are included in the article's Creative Commons licence, unless indicated otherwise in a credit line to the material. If material is not included in the article's Creative Commons licence and your intended use is not permitted by statutory regulation or exceeds the permitted use, you will need to obtain permission directly from the copyright holder. To view a copy of this licence, visit http://creativecommons.org/licenses/by/4.0/ The Creative Commons Public Domain Dedication waiver (http://creativecommons.org/publicdomain/zero/1.0/) applies to the data made available in this article, unless otherwise stated in a credit line to the data. 
Recent estimates suggest that over 700,000 people die annually from antibiotic-resistant infections globally [5]. International agencies have warned that in the absence of a coordinated global response to this epidemic, the death toll from drug-resistant infections could climb to 10 million annually, forcing 24 million people globally into extreme poverty (World Health Organization. New report calls for urgent action to avert antimicrobial resistance crisis. https://www.who.int/news-room/detail/2 9-04-2019-new-report-calls-for-urgent-action-to-avertantimicrobial-resistance-crisis, 2019).

Children are often prescribed antibiotics for potential streptococcal pharyngitis when in fact their signs and symptoms are suggestive of viral upper respiratory tract infections (URTIs) [6, 7]. It has been demonstrated that antibiotic prescribing practices vary by country [8] and by provider specialty [9]. Inappropriate antibiotic use results in increased rates of AMR and increased healthcare utilization due to adverse drug reactions $[10,11]$.

Acute pharyngitis due to Streptococcus pyogenes or group A $\beta$-hemolytic streptococcus (GAS) is diagnosed in up to $37 \%$ of children with pharyngitis [12] and requires antibiotic treatment. This has been traditionally thought of as an infection of school-age children; however, a recent meta-analysis estimated the prevalence of GAS pharyngitis in children under 5 years at $25 \%$, supporting diagnostic testing in this age group when clinically indicated [13].

GAS is a leading global pathogen of infectious diseases [1]. The spectrum of GAS disease extends from superficial infections (pharyngitis, impetigo) to invasive disease (abscesses, cellulitis, sepsis), toxin-mediated manifestations (scarlet fever, toxic shock syndrome, necrotizing fasciitis) and autoimmune sequelae (acute rheumatic fever [ARF], poststreptococcal glomerulonephritis and rheumatic heart disease [RHD]). The most frequent manifestation of GAS infection is pharyngitis, with over 600 million new cases diagnosed per year and complications from invasive GAS disease resulting in 500,000 deaths globally [14].

Timely and targeted antibiotic treatment of GAS infections constitutes the backbone of prevention of complications [15]. This has resulted in a reduction in the burden of RHD in high-income countries; however, high rates of disease persist in low-income countries compounded by lack of healthcare access and unavailability of adequate diagnostic testing [16].

Clinical features and history do not reliably discriminate between GAS and viral pharyngitis, especially in children [17, 18]. International guidelines recommend throat swabs for rapid antigen testing and GAS culture for patients with acute pharyngitis [19] to rapidly detect and target antibiotic treatment for those with GAS infection. The main rationale for antibiotic therapy for GAS pharyngitis is to prevent the development of ARF, the most common cause of acquired heart disease in children. RHD constitutes a substantial burden of chronic disability and death among adolescents and young adults $[15,20]$.

Throat cultures are considered the gold standard for diagnosis of GAS pharyngitis. A recent systematic review demonstrated an $85 \%$ diagnostic sensitivity of SAT among children [21]. However, this sensitivity is suboptimal for rapid assays to be considered stand-alone diagnostic tests, and concurrent back-up throat cultures are required for definitive diagnosis of GAS in children with a negative SAT.

We have previously reported a high diagnostic accuracy of QuickVue ${ }^{\circledast}$ Dipstick Strep A rapid test in a paediatric outpatient clinic setting where high-quality swabs were taken by the attending paediatrician [22]. A similar study in the UAE demonstrated a similarly high diagnostic accuracy of Diaquick Strep A Test [23]. Other studies have also demonstrated a high test sensitivity when throat swabs for SAT are collected by a paediatrician [24-26]. However, these studies' findings are not generalizable to a "real-world" setting where samples are often taken by nurses or non-paediatric physicians, necessitating the need for back-up throat cultures in routine practice to minimize false-negative diagnoses of GAS pharyngitis that would increase the risk of postinfectious sequelae.

In our current study, we evaluated the use of a newer automated rapid streptococcal antigen test system (ASAT) in children presenting with pharyngitis. Standard F strep A antigen test (SD Biosensor, Republic of Korea) is a fluorescence immunoassay with a manufacturerreported test sensitivity of $93.3 \%$ and specificity of $95 \%$ compared to throat culture. We designed a study to determine its diagnostic accuracy in a "real world" scenario in an emergency room where samples are taken by nonpaediatric nurses, and to determine its impact on antibiotic prescribing practices in children presenting with pharyngitis.

\section{Methods}

This was a single-gated diagnostic accuracy study with prospective data collection conducted from March to June 2019 in the emirate of Dubai, United Arab Emirates. Children up to 16 years of age presenting to the emergency department of a multidisciplinary universityaffiliated hospital with signs and symptoms of pharyngitis were included in our study. We excluded children if they were older or if both the automated rapid streptococcal antigen test (A-SAT) and throat culture were not done. Two-hundred and ninety-one children were included in our study. 
All subjects were evaluated and screened for study eligibility by the emergency room (ER) physician. Symptom recording was standardized and all history and physical exam findings were recorded into the hospital's electronic medical record (EMR) by ER physicians. Throat swabs were collected by ER nurses. Patients who had paired throat swabs collected for A-SAT and culture were included in our analysis.

The paired samples were collected with a sterile dry dual red top swab and stored in a clear and dry plastic tube. The samples were transported to the Pathology and Laboratory Department at the Mediclinic City Hospital as soon as possible after collection for testing. This laboratory runs $24 \mathrm{~h}$ and is accredited by the College of American Pathologists (CAP), Joint Commission International (JCI) and holds the ISO-15189 certification. Throat swabs for culture were inoculated as described previously [22].

The A-SAT is an automated fluorescence immunoassay used to detect streptococcal antigen in throat specimens from patients with clinical symptoms. Bacterial antigen is extracted from the patient's swab by the laboratory technician and placed into the sample well of the test device for analysis. Pre-programmed algorithms of the automated device display test results on the device's screen. The measured fluorescence signal is numerically represented by a cut-off index value (COI). A $\mathrm{COI}$ greater than or equal to 1.0 is interpreted as positive, while a COI of less than 1.0 is interpreted as a negative test.

The automated fluorescence immunoassay takes 10-15 $\mathrm{min}$ to perform. However, there is a delay of $30-60 \mathrm{~min}$ between collecting the sample and results being uploaded on the hospital's EMR. Once results are available, the ER physician usually makes antibiotic prescription decisions. Some parents request a prescription and leave pending the A-SAT results. Once results are available, the ER doctor calls and informs the parents and provides guidance on whether or not antibiotics should be started.

In all cases, parents signed a consent form at the time of their child's hospital registration. Collection of throat swabs was done as a part of the child's healthcare process, which is covered by the above general consent. Hence, no separate consent was obtained from parents at the time of sample collection.

This study was reviewed by the Institutional Review Board (IRB) of Mohammed Bin Rashid University of Medicine and Health Sciences (MBRU) Student Research Projects (SRP) committee (MBRU-IRB-SRP-1042017). The data was analyzed using the Statistical Package for Social Sciences (SPSS) 24 software. Our study followed the STARD reporting protocol. Since this was a time-frame study, no power or sample size calculations were required.

\section{Results}

Two hundred and ninety-one children were enrolled in our study. Their mean age was 4.2 years, ranging from 5 months to 16 years. Only 65 (22\%) children were Emiratis, and the rest were expatriates representing various ethnicities all over the world. 187 (64.7\%) children were under the age of 5 years and 168 (57.7\%) were male. Most children had a history of fever (93.4\%), and ten (3.4\%) reported receiving a dose of antibiotics prior to presentation.

Overall, A-SAT was positive in 94 (32.3\%) and negative in 197 (67.6\%) patients, while throat culture was positive in $90(30.9 \%)$ and negative in 201 (69\%) (Fig. 1). There was inconsistency between A-SAT and culture results in six children. A-SAT only missed one child with GAS pharyngitis who tested positive on throat culture (Table 1).

The sensitivity and specificity of A-SAT in the identification of GAS pharyngitis were $98.9 \%$ (95\% CI 98, 100\%) and $97.5 \%$ (95\% CI 95.7, 99.3\%) respectively. A-SAT had a PPV of $94.7 \%$ (95\% CI 92.1, 97.3\%) and NPV of $99.5 \%$ (95\% CI 98.7, 100\%) (Table 2).

Out of a potential 291 antibiotic prescriptions, only 94 children (32\%) were prescribed oral antibiotics for GAS pharyngitis, while the vast majority (68\%) were not. For one child with negative A-SAT and positive throat culture, parents were informed of the result over the phone and antibiotics were initiated.

\section{Discussion}

This is the first study evaluating the accuracy of A-SAT in the Middle East. We have previously reported a high diagnostic accuracy of rapid streptococcal antigen tests, comparable to throat culture, when performed by an attending pediatrician in the outpatient clinic setting in the United Arab Emirates [22, 23]. Unfortunately, those findings were not generalizable to a "real world" scenario where children are often assessed by general physicians and throat samples are taken by non-pediatric healthcare providers. Studies have reported variation in antigen test performance by inoculum size and provider characteristics, resulting in lower test sensitivity attributable to suboptimal sampling techniques and provider experience in obtaining samples from children $[25,26]$.

In this study, we have demonstrated a very high diagnostic efficacy of A-SAT, with NPV approaching 100\% in children presenting to the ER with signs and symptoms of pharyngitis. This shows that new-generation rapid streptococcal antigen assays that utilize fluorescent immunoassay technology, are robust to variations in sampling technique and perform well in real-world clinical scenarios, making them very useful in providing rapid and accurate diagnosis of GAS pharyngitis in children. 


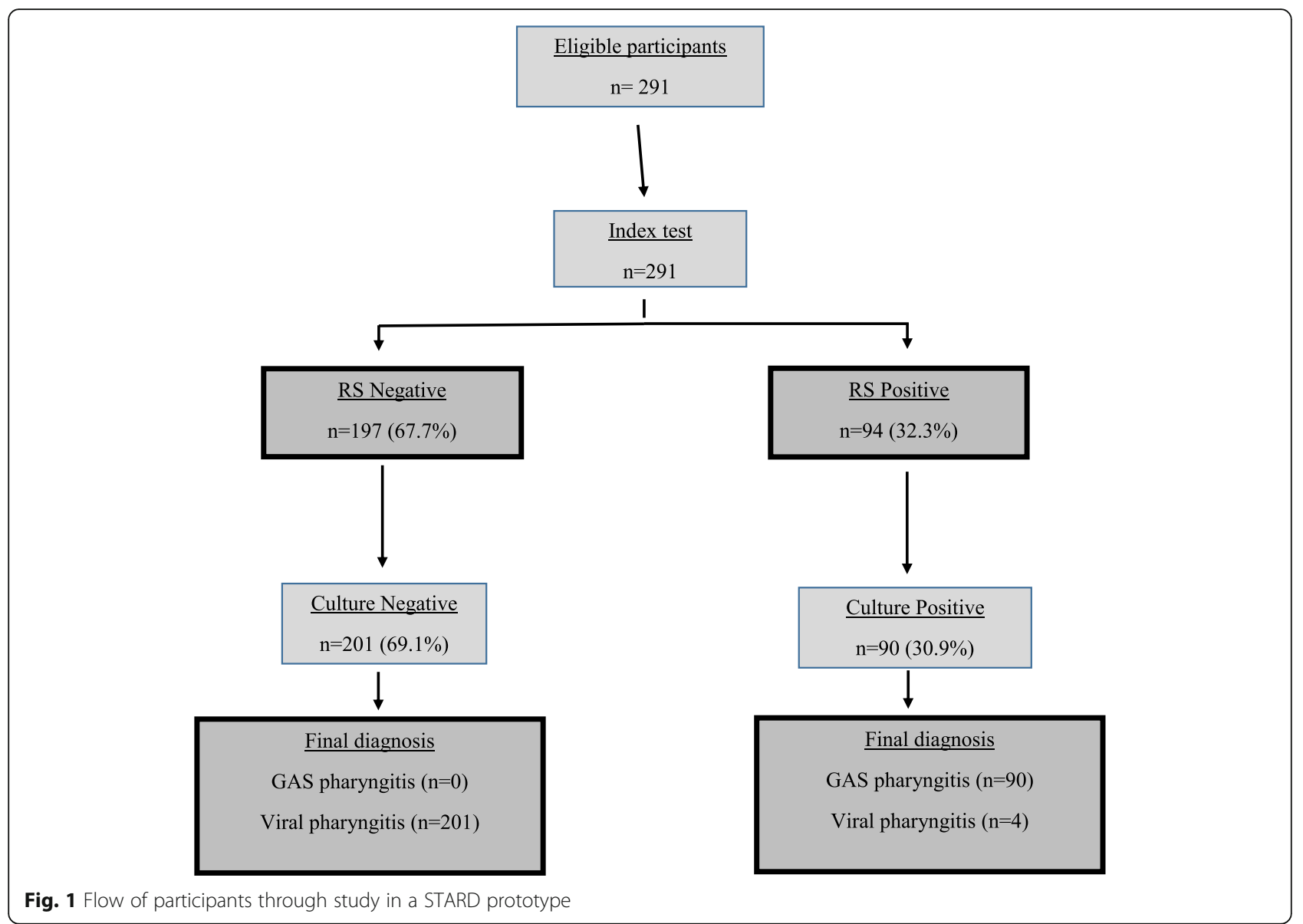

As expected, most children had viral illnesses contributing to their symptoms, as confirmed by negative throat cultures in $69 \%$ of our study cohort. Since our study objective was to examine the diagnostic accuracy of A-SAT in comparison to throat culture, we decided to include 10 children who reported receiving antibiotics prior to presentation; all of them had negative A-SAT and throat cultures.

Among our patients, the prevalence of GAS pharyngitis was $30.9 \%$. This is higher than the previously reported prevalence of $14 \%$ between 2004 and 2006 [23] and 20.6\% between 2016 and 2018 [22] among children in the United Arab Emirates. The increase in reported prevalence in our current study could represent a referral bias of sicker

Table 1 Automated rapid streptococcal antigen test (A-SAT) and throat culture results $(n=291)$

\begin{tabular}{lllll}
\hline & & A-SAT & & \\
& & Negative & Positive & Total \\
Culture & Negative & 196 & 5 & 201 \\
& Positive & 1 & 89 & 90 \\
Total & & 197 & 94 & 291 \\
\hline
\end{tabular}

children presenting to the ER rather than to an outpatient clinic. These figures are consistent with the reported prevalence of GAS pharyngitis ranging between 30 and $37 \%$ in other pediatric studies $[13,21]$.

Our study was conducted in the emirate of Dubai in the United Arab Emirates, which is home to people from over 200 countries. Expatriates constitute 80\% of the population of Dubai, home to one of the world's largest percentage of immigrants. Our patient cohort was highly representative of Dubai's population mix, with expatriates constituting around $78 \%$ of the study population. Hence, our study findings are likely generalizable to children from various ethnicities around the world.

Table 2 Clinical performance of automated rapid Strep A test (A-SAT) when compared to throat culture for the detection of Group A Streptococcus pharyngitis $(n=291)$

\begin{tabular}{ll}
\hline & A-SAT \\
\hline Sensitivity (95\% Cl) & $98.9 \%(98-100)$ \\
Specificity (95\% Cl) & $97.5 \%(95.7-99.3)$ \\
PPV (95\% Cl) & $94.7 \%(92.1-97.3)$ \\
NPV (95\% Cl) & $99.5 \%(98.7-100)$ \\
\hline
\end{tabular}


The current recommendations of throat cultures as a confirmatory test on patients with a negative rapid test [19] is not achievable in most low-income countries due to the prohibitive cost. Ehrlich et al. have reported that follow-up throat cultures for negative rapid tests would detect 21 additional cases of rheumatic heart disease at a societal cost of an additional $\$ 8$ million per case prevented in the United States [27]. Among South African children, obtaining throat cultures in all children presenting with pharyngitis as a primary prevention strategy for RHD is prohibitively expensive; treating all such children with intramuscular penicillin is the most cost-effective strategy [15] but risks the rapid development of AMR. The high sensitivity and NPV of A-SAT in our study suggests that this can be used as a stand-alone test, without the routine need for confirmatory throat cultures, striking a balance between reducing costs and preventing excessive use of antibiotics especially in resource-lacking countries. However, it needs further review before this strategy can be adopted into routine clinical practice.

Utilizing A-SAT testing provided rapid and accurate results, resulting in a striking reduction in antibiotic prescriptions among our cohort. The vast majority $(n=197$, $68 \%$ ) of children in our study were not prescribed antibiotics for acute pharyngitis. Fewer antibiotic prescriptions result in direct monetary savings, reduction in antibiotic use, and decreased emergence of resistant pathogens in our community. This reduction in antibiotic use also helps to reduce the clinical and economic burden of adverse drug reactions and unnecessary healthcare utilization due to these effects $[10,11]$.

The main strength of our study was that it was conducted in the busy ER of a tertiary care hospital with a diverse patient population from various ethnicities. This represents a real-world scenario and increases the applicability of our findings to other clinical settings. The automation of A-SAT minimizes false positive results that can result from prolonged incubation times when tests are performed manually. This likely improved the diagnostic accuracy of A-SAT in our study. In addition, all patients underwent A-SAT and throat culture on paired swabs collected simultaneously, hence limiting differential verification bias. Paired swabs also ensured that there was no delay in sample testing, which could lead to over- or underestimation of results. The major limitation of our study is that it represents a convenience sample of patients presenting to the ER of a single multidisciplinary hospital rather than an array of hospitals, hence limiting its generalizability to other clinical settings.

\section{Conclusions}

This is the first study to demonstrate that the diagnostic accuracy of an automated rapid Strep A test among children in an emergency room setting in the Middle East is comparable to that of throat culture. We have demonstrated that the A-SAT is a simple, rapid, and highly reliable test which can help reduce unnecessary antibiotic prescriptions in children presenting with pharyngitis, and hence limit the spread of antimicrobial resistance.

\begin{abstract}
Abbreviations
GAS: Group A $\beta$ - haemolytic Streptococcus; SAT: Rapid streptococcal A antigen test; AMR: Antimicrobial resistance; A-SAT: Automated rapid streptococcal A antigen test; PPV: Positive predictive value; NPV: Negative predictive value; URTI: Upper respiratory tract infections; ARF: Acute rheumatic fever; RHD: Rheumatic heart disease; ER: Emergency Room; EMR: Electronic medical record; CAP: College of American Pathologists; JCl: Joint Commission International; COI: Cut-off index; IRB: Institutional Review Board; MBRU: Mohammed Bin Rashid University of Medicine and Health Sciences; SRP: Student Research Projects; SPSS: Statistical Package for Social Sciences
\end{abstract}

\section{Acknowledgements}

We gratefully acknowledge Ms. Hima Jayakrishnan, microbiology technician at Mediclinic City Hospital, and Mr. Mohammed Zaki, medical student at Mohammed Bin Rashid University, for their assistance.

\section{Authors' contributions}

SH collected data and drafted the manuscript. ZM collected data, drafted and critically reviewed the manuscript. AK performed statistical analysis and drafted the manuscript. FA conceived the study, collected data, and drafted the manuscript. All authors read and approved the final manuscript.

\section{Funding}

This research did not receive any specific grant from funding agencies in the public, commercial, or not-for-profit sectors.

\section{Availability of data and materials}

Data which supports the findings of this study is available from Mediclinic City Hospital. However, restrictions apply to the availability of this data, which was used under license for the current study and is not publicly available. Study data can be made available upon reasonable request from the authors and with permission of Mediclinic City Hospital, Dubai, UAE.

\section{Ethics approval and consent to participate}

This study was approved by the Mohammed Bin Rashid University of Medicine and Health Sciences (MBRU)-IRB Student Research Projects (SRP) committee (MBRU-IRB-SRP-104-2017). In all cases, parents signed a consent form at the time of their child's hospital registration. The collection of paired swabs constitutes a part of the child's healthcare process, which is covered by the above general consent. Hence, no separate consent was sought from parents at the time of sample collection.

\section{Consent for publication}

Not applicable.

\section{Competing interests}

All authors have no conflicts to declare.

\section{Author details}

${ }^{1}$ College of Medicine, Mohamed Bin Rashid University of Medicine and Health Sciences, Dubai Healthcare City, Building 14, Dubai 505005, United Arab Emirates. ${ }^{2}$ Department of Pediatrics, Mediclinic City Hospital. Dubai Healthcare City, Building 37, Dubai 505004, United Arab Emirates. ${ }^{3}$ Pediatric Infectious Diseases, Mediclinic City Hospital. Dubai Healthcare City, Building 37, Dubai 505004, United Arab Emirates. ${ }^{4}$ Department of Biostatistics, Mohamed Bin Rashid University of Medicine and Health Sciences. Dubai Healthcare City, Building 14, Dubai 505005, United Arab Emirates. 
Received: 1 September 2020 Accepted: 20 January 2021

Published online: 25 January 2021

\section{References}

1. Vekemans J, Gouvea-Reis F, ... JK-Cl, 2019 undefined. The Path to Group A Streptococcus Vaccines: World Health Organization Research and Development Technology Roadmap and Preferred Product Characteristics. academic.oup.com. [cited 2020 Jul 13]; Available from: https://academic.oup. com/cid/article-abstract/69/5/877/5280612

2. de Kraker MEA, Davey PG, Grundmann H. Mortality and hospital stay associated with resistant Staphylococcus aureus and Escherichia coli bacteremia: Estimating the burden of antibiotic resistance in Europe. PLoS Med. 2011;8(10) [cited 2020 Jul 13] Available from: /pmc/articles/ PMC3191157/?report=abstract.

3. Stewardson AJ, Allignol A, Beyersmann J, Graves N, Schumacher M, Meyer R, et al. The health and economic burden of bloodstream infections caused by antimicrobial-susceptible and non-susceptible Enterobacteriaceae and Staphylococcus aureus in European hospitals, 2010 and 2011: A multicentre retrospective cohort study. Eurosurveillance. 2016;21(33) [cited 2020 Jul 13] Available from: /pmc/articles/PMC4998424/?report=abstract

4. Kostyanev T, Bonten MJM, O'brien S, Steel H, Ross S, François B, et al. The Innovative Medicines Initiative's New Drugs for Bad Bugs programme: European public-private partnerships for the development of new strategies to tackle antibiotic resistance. [cited 2020 Jul 13]; Available from: www. prepare-europe.eu

5. WHO. Antimicrobial resistance. Global report on surveillance. World Heal Organ. 2014;61(3):12-28.

6. Hersh AL, Fleming-Dutra KE, Shapiro DJ, Hyun DY, Hicks LA. Frequency of first-line antibiotic selection among us ambulatory care visits for Otitis media, sinusitis, and pharyngitis. JAMA Intern Med. 2016;176:1870-2.

7. Havers FP, Hicks LA, Chung JR, Gaglani M, Murthy K, Zimmerman RK, et al. Outpatient antibiotic prescribing for acute respiratory infections during influenza seasons. JAMA Netw Open. 2018;1(2):e180243.

8. Tyrstrup M, van der Velden A, Engstrom S. Antibiotic prescribing in relation to diagnoses and consultation rates in Belgium, the Netherlands and Sweden: use of European quality indicators. Scand J Prim Heal Care. 2017; 35:10-8.

9. Frost $\mathrm{HM}, \mathrm{McLean} H \mathrm{HQ}$, Chow B. Variability in antibiotic prescribing for upper respiratory illnesses by provider specialty. J Pediatr. 2018;203:76-85.e8.

10. Sultana J, Cutroneo P, Trifirò G. Clinical and economic burden of adverse drug reactions. J Pharmacol Pharmacother. 2013;4(Suppl 1):S73-7.

11. Schneider JE, Boehme C, Borisch B, Dittrich S. Application of a simple pointof-care test to reduce UK healthcare costs and adverse events in outpatient acute respiratory infections. J Med Econ. 2020;23(7):673-82 [cited 2020 Jul 15] Available from: https://www.tandfonline.com/doi/full/10.1080/13696 998.2020.1736872.

12. Danchin MH, Rogers S, Kelpie L, Selvaraj G, Curtis N, Carlin JB, et al. Burden of acute sore throat and group A streptococcal pharyngitis in school-aged children and their families in Australia. Pediatrics. 2007;120(5):950-7 [cited 2020 Jul 13] Available from: https://pubmed.ncbi.nlm.nih.gov/17974731/.

13. Shaikh N, Leonard E, Pediatrics JM. Prevalence of streptococcal pharyngitis and streptococcal carriage in children: a meta-analysis. Am Acad Pediatr. 2010; [cited $2020 \mathrm{Jul}$ 13]; Available from: https://pediatrics.aappublications. org/content/126/3/e557.abstract.

14. Carapetis JR, Steer AC, Mulholland EK, Weber M. The global burden of group A streptococcal diseases. Lancet Infect Dis. 2005;5(11):685-94.

15. Irlam J, Mayosi BM, Engel M, Gaziano TA. Primary prevention of acute rheumatic fever and rheumatic heart disease with penicillin in south African children with pharyngitis: a cost-effectiveness analysis. Circ Cardiovasc Qual Outcomes. 2013;6(3):343-51.

16. Watkins DA, Johnson CO, Colquhoun SM, Karthikeyan G, Beaton A, Bukhman G, et al. Global, regional, \& national burden of rheumatic heart disease, 1990-2015. N Engl J Med. 2017:377(8):713-22.

17. Lin MH, Fong WK, Chang PF, Yen CW, Hung KL, Lin SJ. Predictive value of clinical features in differentiating group A Bhemolytic streptococcal pharyngitis in children. J Microbiol Immunol Infect. 2003;36(1):21-5.

18. Roggen I, van Berlaer G, Gordts F, Pierard D, Hubloue I. Centor criteria in children in a paediatric emergency department: for what it is worth. BMJ Open. 2013;3(4):e002712. [cited 2020 Jul 13] Available from: http://bmjopen. bmj.com/lookup/. https://doi.org/10.1136/bmjopen-2013-002712.
19. Shulman ST, Bisno AL, Clegg HW, Gerber MA, Kaplan EL, Lee G, et al. Clinical Practice Guideline for the Diagnosis and Management of Group A Streptococcal Pharyngitis: 2012 Update by the Infectious Diseases Society of America. 2012 [cited 2020 Jul 14]; Available from: https://academic.oup. com/cid/article-abstract/55/10/e86/321183

20. Omurzakova NA, Yamano Y, Saatova GM, Mirzakhanova MI, Shukurova SM, Kydyralieva RB, et al. High incidence of rheumatic fever and Rheumatic heart disease in the republics of Central Asia. Int J Rheum Dis. 2009;12(2): 79-83.

21. Cohen JF, Bertille N, Cohen R, Chalumeau M. Rapid antigen detection test for group A streptococcus in children with pharyngitis. Cochrane Database Syst Rev. 2016;2016 [cited 2020 Jul 13]. Available from: /pmc/articles/ PMC6457926/?report=abstract.

22. Safizadeh Shabestari SA, Malik ZA, Al-Najjar FYA. Diagnostic accuracy of QuickVue ${ }^{\circledast}$ Dipstick Strep A test and its effect on antibiotic prescribing in children in the United Arab Emirates. BMC Pediatr. 2019;19(1) [cited 2020 Jul 13] Available from: /pmc/articles/PMC6844028/?report=abstract.

23. FYA A-N, Uduman SA. Clinical utility of a new rapid test for the detection of group A Streptococcus and discriminate use of antibiotics for bacterial pharyngitis in an outpatient setting. Int J Infect Dis. 2008;12(3):308-11 [cited 2020 Jul 13] Available from: http://intl.elsevierhealth.com/journals/ijid.

24. Kurtz B, Kurtz M, Roe M, Todd J. Importance of Inoculum Size and Sampling Effect in Rapid Antigen Detection of S. pyogenes Pharyngitis. J Clin Microbiol. 2000;38(1):279-81.

25. Fox JW, Cohen DM, Marcon MJ, Cotton WH, Bonsu BK. Performance of Rapid Streptococcal Antigen Testing Varies by Personnel. J Clin Microbiol. 2006;44(11):3918-22 [cited 2020 Jul 14] Available from: http://jcm.asm.org/.

26. Cohen JF, Chalumeau M, Levy C, Bidet P, Benani M, Koskas M, et al. Effect of clinical spectrum, inoculum size and physician characteristics on sensitivity of a rapid antigen detection test for group a streptococcal pharyngitis. Eur J Clin Microbiol Infect Dis. 2013:787-93.

27. Ehrlich JE, Demopoulos BP, Daniel KR, Ricarte MC, Glied S. Cost-effectiveness of treatment options for prevention of rheumatic heart disease from group A streptococcal pharyngitis in a pediatric population. Prev Med (Baltim). 2002;35(3):250-7.

\section{Publisher's Note}

Springer Nature remains neutral with regard to jurisdictional claims in published maps and institutional affiliations.

Ready to submit your research? Choose BMC and benefit from:

- fast, convenient online submission

- thorough peer review by experienced researchers in your field

- rapid publication on acceptance

- support for research data, including large and complex data types

- gold Open Access which fosters wider collaboration and increased citations

- maximum visibility for your research: over $100 \mathrm{M}$ website views per year

At $\mathrm{BMC}$, research is always in progress.

Learn more biomedcentral.com/submissions 